\title{
Cognitive Pilot Channel Enabling Spectrum Awareness
}

\author{
O. Sallent, J. Pérez-Romero, R. Agustí \\ Dept. Signal Theory and Communications \\ Universitat Politècnica de Catalunya (UPC). Barcelona, Spain \\ E-mail: [sallent, jorperez, ramon]@tsc.upc.edu
}

\author{
P. Cordier \\ France Telecom Research \&Development, \\ Issy-Les-Moulineaux, France \\ E-mail: pascal.cordier@orange-ftgroup.com
}

\begin{abstract}
This paper presents the Cognitive Pilot Channel (CPC) solution as a mechanism allowing the terminal to be aware of the communication means available at a given time and place in a flexible spectrum management scenario. The different aspects to be considered in the CPC deployment are highlighted, together with the different implementation options. As a result, a CPC dimensioning methodology is formulated, identifying the main parameters involved in the process and the corresponding design trade-offs.
\end{abstract}

Keywords-Cognitive Pilot Channel (CPC), Flexible Spectrum Management, Spectrum awareness.

\section{INTRODUCTION}

The current trend in radio communication systems is towards a flexible spectrum management that, in its broadest meaning, aims at making the best possible and most efficient use of spectrum resource available to mobile Radio Access Technologies (RATs) by exploiting the dynamic varying nature of the traffic as well as of the demand and dynamically assigning the spectrum to meet the required demand with limited available resources [1]. Spectrum refarming, which allows a specified frequency band to be available for a different kind of usage or technology (e.g. refarming of GSM spectrum for UMTS/HSXPA), can be seen as the simplest use case for flexible spectrum management. Another example is the so-called digital dividend, where the frequencies in the UHF band cleared by the transition of analog to digital television could be utilized by mobile TV, cellular technologies like UMTS, LTE, WiMAX and also for flexibly sharing spectrum between smart radio technologies. In this respect, the proposition of the TV band Notice of Proposed Rule Making (NPRM) [2], allowing unlicensed radios to operate in the TV broadcast bands if no harmful interference is caused to incumbent services (i.e. TV receivers), was a first milestone allowing dynamic spectrum access mechanisms.

Considering a flexible spectrum management framework, spectrum awareness arises as a basic challenge in a generic scenario, where a number of transceivers with flexible timevarying assignment of operating frequency and/or RAT are deployed. Spectrum awareness from the mobile's perspective refers to the mechanisms allowing the terminal to be aware of the communication means available at a given time and place.

In order to get knowledge of its radio environment, the mobile terminal may sense some parts of the spectrum, but this may result in a very time- and power-consuming operation if the spectrum bands to be sensed are too large. As an alternative, following a similar approach as the Spectrum Information Channel in [3] and the CSCC in [4], the Cognitive Pilot Channel (CPC) concept was conceived as a solution in conveying the necessary information from the network side to let the terminal know e.g. the available frequency bands, RATs, operators, etc. at a given time and place [5][6][7]. The CPC concept is related to a specific phase of the cognition cycle, consisting in observing the environment. More recently, the use cases for a CPC-like approach have been further developed and extended within IEEE P1900.4 and include e.g. the provision from the network side of radio resource selection policies that will guide the terminals in their own reconfiguration decisions [8].

Regarding the regulatory framework related to the implementation of cognitive radio systems and introduction of flexible spectrum management, actions have already been initiated within ITU-R WP 5A with inputs from European Projects $E^{2} R$ and $E^{3}$, contributing to the inclusion of the CPC concept in the working document on Cognitive Radio Systems (CRS), focusing on the radio environment discovery at switch on of the mobile terminal. Additional standardization activities have also been launched within ETSI and CPC prestandardization is currently under development in the framework of the ETSI RRS (Reconfigurable Radio Systems) Technical Committee.

Based on all the above, this paper focuses on the CPC solution for the spectrum awareness problem. Different implementation alternatives are analyzed and the corresponding pros and cons are highlighted with the support of performance evaluation metrics.

The rest of the paper is organized as follows. Section II presents different alternatives and aspects to be taken into account when designing and deploying the $\mathrm{CPC}$, and provides a formulation to compute the required CPC capacity. Section III illustrates the effect of the different parameters and alternatives with results obtained in different scenarios. Finally, conclusions are summarized in Section IV.

\section{CPC DESIGN AND DEPLOYMENT}

Let consider the illustrative scenario depicted in Figure 1, where a number of transmitters, with possibly time-varying assignment of RATs and operating frequencies, are deployed. Furthermore, in its more general view, a stand-alone CPC 
transmitter separated from the rest of transmitters in the scenario is deployed. The CPC transmitter, which is physically realized on a given RAT, operating frequency and associated bandwidth, is in charge to convey spectrum awareness information related to its coverage area.

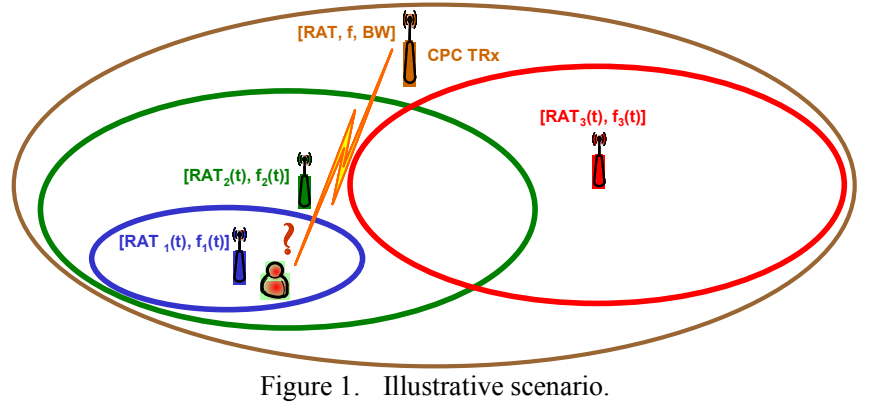

The different aspects to be considered when moving from $\mathrm{CPC}$ concept to implementation, regarding the radio part, can be identified:

- CPC physical and link layer specification. This includes e.g. the definition of the appropriate RAT to carry CPC information, either a new one or the adaptation of a legacy one, the required bandwidth to transmit the information to be conveyed and considerations on the operating frequency.

- CPC deployment. This includes the definition of the number of CPC transceivers (TRx) with their corresponding configuration (e.g. power levels, etc.) necessary to provide the CPC information to the mobile terminals on a certain geographical region.

It is worth noting that, although business model considerations are out of the scope of this paper, the perspective provided here is generic enough to be applied to different business models regarding the CPC operation and exploitation (e.g. CPC is deployed by a CPC-operator with its own deployed infrastructure to convey information from different access providers; CPC is operated by a legacy cellular operator who exploits already existing sites to convey information for an association of operators; etc.).

As for the RAT used for CPC, two main options are identified:

- In-band CPC. CPC information is conveyed using logical channels of the same RATs that are used for the user data transmission.

- Out-band CPC. The CPC information is conveyed using a CPC-specific RAT, separate from that used for user data transmission.

As for the information delivery mode, two main options are identified [7]:

- Broadcast CPC. This strategy only uses a downlink broadcast channel, where the information is periodically and continuously transmitted.
- On-demand CPC. In this case, the information is transmitted only when needed and requested by a terminal. Therefore, the on-demand $\mathrm{CPC}$ requires both the uplink and downlink components.

As for the CPC operating frequency, a number of alternatives can be envisaged. Some possibilities are:

- It is fixed and harmonized at global/regional basis, consortium of access providers basis or only at internal level within a given access provider domain.

- $\quad$ It is neither harmonized nor fixed (i.e. CPC operating frequency may change). Note that in this case CPC does not support switch on use case (i.e. switch on relies on scanning).

As for the information to be conveyed by the CPC, the following aspects need to be noted:

- The amount of information will depend on the complexity of the scenario (in terms of resulting regions with radioelectrical commonalities) within the $\mathrm{CPC}$ transmitter coverage range.

- The amount of information will depend on the information structuring scheme used to characterize the scenario. In particular, some possibilities to structure the information could be:

- Single layer. In this case the CPC provides information on a one-by-one RAT/frequency spatial availability basis. For example, for the scenario depicted in Figure 2, CPC informs about 3 different regions: (1) where RAT1 is available, (2) where RAT2 is available and (3) where RAT3 is available.

○ Multiple layer. In this case the CPC provides information on every region where a different combination of RATs/frequencies is available. For example, for the scenario depicted in Figure 2, CPC informs about 4 different regions: (1) where coverage from RAT2 and RAT1 is available, (2) where only RAT2 is available, (3) where only RAT3 is available and (4) where RAT2 and RAT3 are available.

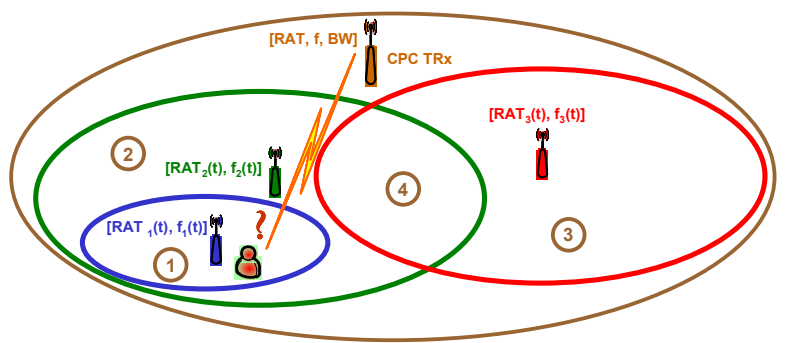

Figure 2. Identification of regions in the scenario.

At this stage, it is worth remarking that knowing the position of the mobile terminal is not a strict requirement for the CPC operation but a capability that enables higher 
efficiency in gaining spectrum awareness:

- In case positioning is not available, as long as the mobile terminal is able to detect (for the Broadcast CPC delivery mode) or contact (for the On-demand CPC delivery mode) the CPC transceiver, CPC is aware that the terminal is within its coverage area and the information about the different regions in that area can be provided. In the worst case, the mobile terminal has to make a scanning over the possibilities indicated by CPC to find out what RATs and frequencies are available at its actual position.

- In case positioning is available, CPC can readily provide the availability of RATs and frequencies at the actual position.

In the following subsections, the different elements involved in the CPC design and dimensioning process are further developed.

\section{A. Offered CPC capacity}

The CPC radio interface, either being a newly defined access technology or the adaptation of an existing one, will be operating at a certain frequency $f$ and occupying a certain bandwidth $B W$. The physical and link layer design will end up with a certain capacity to convey information through the CPC from every single CPC transceiver (TRx), denoted here as $R$ bits/s/TRx.

The CPC can be deployed in different forms. In its broadest perspective, there could be specifically deployed infrastructure to implement CPC transceivers. Clearly, reuse of already existing infrastructure can be very attractive to reduce investment. In that case, a number of possibilities also arise. For example, CPC information could be transmitted over every cell site in a cellular-based network or only over a subset of the sites. Similarly, the CPC information could be transmitted from broadcasting towers.

Whatever the deployment strategy is, it will be characterized here by a certain density of CPC transceivers over a given geographical area, $T \mathrm{TRx} / \mathrm{km}^{2}$. Therefore, given a certain $\mathrm{CPC}$ radio interface and a CPC deployment strategy, the resulting capacity is $R \times T$ bits $/ \mathrm{s} / \mathrm{km}^{2}$.

\section{B. Demanded CPC capacity}

Let denote by $X$ zones $/ \mathrm{km}^{2}$ the density of zones to be signaled by the CPC. Let denote by $M$ bits/zone the number of bits needed to represent a single zone. The required CPC capacity depends on the delivery mode approach followed:

- For a Broadcast mode, information about the operators/RATs/frequencies available within the range of a CPC transceiver is provided continuously and periodically. Fixing a periodicity of $P$ seconds, which is the maximum delay that is allowed for the terminal to receive the information through the broadcast CPC, the required CPC capacity is given by $M \times X / P$ bits $/ \mathrm{s} / \mathrm{km}^{2}$.
- For an On-demand mode, information about the operators/RATs/frequencies available within the range of a $\mathrm{CPC}$ transceiver is provided upon request. Denoting the density of requests by $L$ requests $/ \mathrm{s} / \mathrm{km}^{2}$ and given that a request generates the need to specify the radio conditions in a given zone, the required CPC capacity is given by $M \times L$ bits $/ \mathrm{s} / \mathrm{km}^{2}$.

\section{CPC dimensioning}

Table I summarizes the parameters involved in the $\mathrm{CPC}$ design and deployment process. A proper dimensioning will match the demanded CPC capacity with the offered CPC capacity. Therefore, the balance must be achieved among the following parameters:

- $\quad$ For Broadcast CPC, $R \times T=M \times X / P$.

- For On-demand CPC, $R \times T=M \times L$.

Note that the comparison between Broadcast and Ondemand delivery modes is established as follows:

- Broadcast CPC compares favorably to On-demand CPC provided that $X / P<L$.

- $\quad$ On-demand CPC compares favorably to Broadcast CPC provided that $X / P>L$.

TABLE I. LIST OF PARAMETERS INVOLVED IN THE CPC DESIGN AND DEPLOYMENT PROCESS.

\begin{tabular}{|l|l|}
\hline Operating frequency & $F$ \\
\hline Bandwidth & $B W$ \\
\hline CPC capacity per transceiver & $R$ bits $/ \mathrm{s} / \mathrm{TRx}$ \\
\hline Density of CPC transceivers & $T$ TRx $/ \mathrm{km}^{2}$ \\
\hline CPC offered capacity & $R \times T$ bits $/ \mathrm{s} / \mathrm{km}^{2}$ \\
\hline $\begin{array}{l}\text { Density of zones with radioelectrical } \\
\text { commonalities }\end{array}$ & $X$ zones $/ \mathrm{km}^{2}$ \\
\hline Bits needed to represent a zone & $M$ bits $/$ zone \\
\hline Broadcast CPC periodicity & $P$ s \\
\hline $\begin{array}{l}\text { CPC demanded capacity for } \\
\text { Broadcast mode }\end{array}$ & $M \times X / P$ bits $/ \mathrm{s} / \mathrm{km}^{2}$ \\
\hline On-demand CPC requests & $L$ requests $/ \mathrm{s} / \mathrm{km}^{2}$ \\
\hline $\begin{array}{l}\text { CPC demanded capacity for On- } \\
\text { demand mode }\end{array}$ & $M \times L$ bits $/ \mathrm{s} / \mathrm{km}^{2}$ \\
\hline
\end{tabular}

It is worth mentioning that, in a non-homogeneous scenario with different RATs deployed in a non-regular pattern, the offered and demanded CPC capacities presented above would represent the average values across the scenario, which may suffice for an estimation of the $\mathrm{CPC}$ requirements.

\section{RESULTS}

\section{A. Reference scenarios and complexity analysis}

The analysis of the scenario complexity in terms of the average density of zones per $\mathrm{km}^{2}$ existing in a given deployment is carried out using the image processing methodology presented in [9]. It consists in digitalizing a scenario building an image whose pixel intensities depend on the detected frequencies in each point.

In order to compare the different CPC approaches, some illustrative scenarios as a benchmarking basis will be considered. In particular, assuming a scenario with two RATs, 
one being a cellular technology with cell radius $R_{C}$ covering the total surface and the other one being composed by a number of isolated hotspots with radius $R_{H}$ and transmitter density $\rho_{T x}$ transmitters $/ \mathrm{km}^{2}$, we will denote as $X_{m}$ the average density of zones per $\mathrm{km}^{2}$ for the multiple layer case, whereas $X_{S}$ represents the single layer case (indeed, $X_{s}$ will be simply given by the sum of cellular and hotspot transmitters' density). The reference scenarios are the following:

- Scenario 1: A cellular technology with radius $R_{C}=1 \mathrm{~km}$ is deployed in the whole area while a density of hotspot transmitters $\rho_{T x}=0.18$ transmitters $/ \mathrm{km}^{2}$, each with radius $R_{H}=200 \mathrm{~m}$ is scattered randomly in the scenario. The total size is $10 \mathrm{~km} \times 10 \mathrm{~km}$. In this case, the resulting number of zones, following a multiple layer approach, is $X_{m 1}=2.54 \mathrm{zones} / \mathrm{km}^{2}$. On the contrary, in the single layer approach the resulting number of zones would be $X_{s 1}=0.6 \mathrm{zones} / \mathrm{km}^{2}$. Figure 3 plots the corresponding image representing the geographical regions in scenario 1. In the figure each color is associated with a combination of frequencies detected in the corresponding point (i.e. an area with a certain color means that the same frequencies are detected in the whole area). In this particular example four frequencies are considered (three for the cellular and one for the hotspots), meaning that there are up to 16 combinations of them that can be detected (i.e. 16 colors).

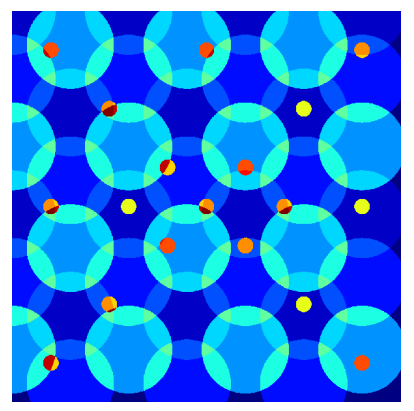

Figure 3. Image representing the regions in scenario 1

- Scenario 2: A cellular technology with radius $R_{C}=1 \mathrm{~km}$ is deployed in the whole area while a density of hotspot transmitters $\rho_{T x}=0.18$ transmitters $/ \mathrm{km}^{2}$, each with radius $R_{H}=700 \mathrm{~m}$ is scattered in the scenario. In this case, it results $X_{m 2}=3.64 \mathrm{zones} / \mathrm{km}^{2}$ in the case of the multiple layer approach and $X_{s 2}=0.6$ zones $/ \mathrm{km}^{2}$ in the case of the single layer approach (i.e. the same as in scenario 1 since in fact the total number of transmitters is the same). Figure 4 plots the corresponding image associated to this scenario.

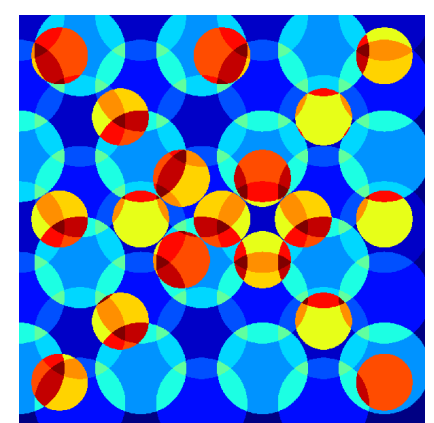

Figure 4. Image representing the regions in scenario 2
- Scenario 3: A cellular technology with radius $R_{C}=200 \mathrm{~m}$ is deployed in the whole area while a density of hotspot transmitters $\rho_{T x}=0.18$ transmitters $/ \mathrm{km}^{2}$, each with radius $R_{H}=200 \mathrm{~m}$ is scattered in the scenario. In this case, it results $X_{m 3}=54.71$ zones $/ \mathrm{km}^{2}$ and $X_{s 3}=10.04$ zones $/ \mathrm{km}^{2}$. The scenario is plot in Figure 5.

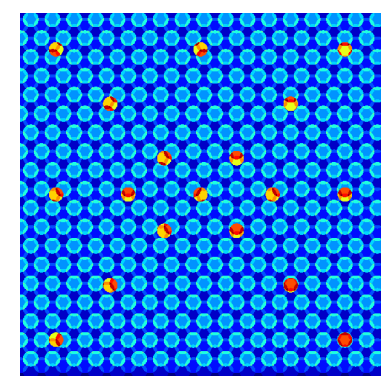

Figure 5. Image representing the regions in scenario 3.

\section{B. Analysis of the demanded CPC capacity}

Figure 6, Figure 7 and Figure 8 plot the corresponding CPC demanded capacity for the broadcast CPC in the three considered scenarios for different values of periodicity $P$ and number of bits per zone $M$ in the multiple layer case, i.e. considering $X=X_{m}$. Clearly, scenario 3 puts the strongest requirements in terms of capacity since the number of zones to be signaled by CPC is higher. Specifically, the required capacity is more than ten times larger than with scenarios 1 and 2. As for the comparison between scenario 1 and 2, the requested capacity in scenario 2 is higher because the larger coverage area of the hotspots generates a larger number of homogeneous areas when intersecting with the cellular coverage. In turn, Figure 9 plots the corresponding capacity for the on-demand case, which is valid for all three scenarios, because it is not sensitive to the density of zones. In this case, the requested capacity increases linearly with the ratio of user requests $L$. Notice that, for the considered range of values of $L$, the requested capacity in scenario 3 can be significantly reduced with the on-demand approach, particularly when the periodicity $P$ of the broadcast CPC is small.

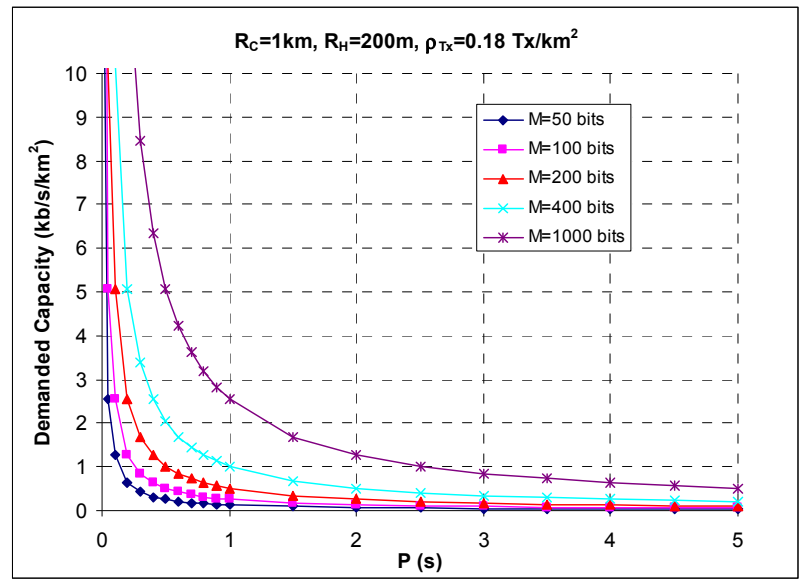

Figure 6. CPC demanded capacity for the broadcast CPC in scenario 1 as a function of $P$ and for different values of $M$, for the multiple layer case. 


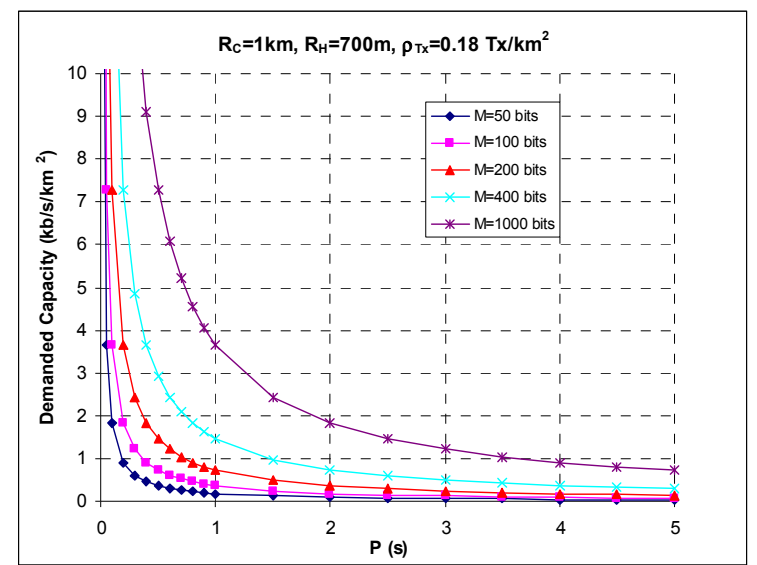

Figure 7. CPC demanded capacity for the broadcast $\mathrm{CPC}$ in scenario 2 as a function of $P$ and different values of $M$, for the multiple layer case.

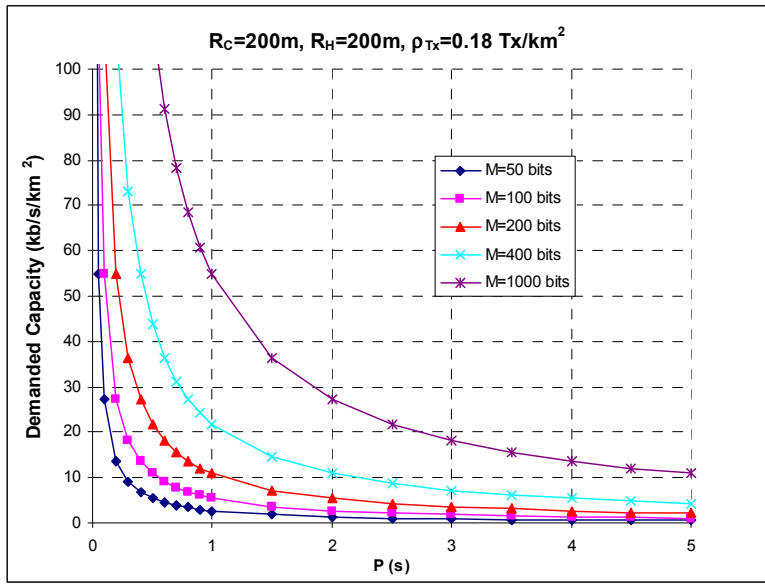

Figure 8. CPC demanded capacity for the broadcast CPC in scenario 3 as a function of $P$ and different values of $M$, for the multiple layer case.

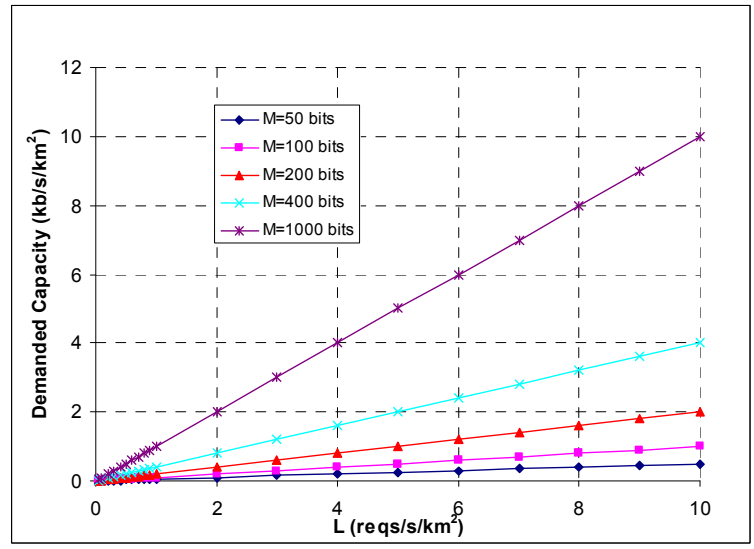

Figure 9. CPC demanded capacity for the on-demand CPC as a function of the ratio of user requests $L$ and different values of $M$ in scenarios 1, 2 and 3 .

Figure 10 and Figure 11 plot the comparison between the single layer coding $\left(X=X_{s}\right)$ and the multiple layer coding $\left(X=X_{m}\right)$ for the broadcast CPC in scenarios 1 and 3, respectively, for different numbers of bits per zone $M$. It can be observed that, for a given value of $M$, as long as the single layer reduces the number of regions $/ \mathrm{km}^{2}$ to be transmitted, the demanded capacity is reduced with respect to the multiple layer case. In addition, it is also worth mentioning that the value of $M$ itself would be dependant on whether the single layer or the multiple layer coding is used. Specifically with the multiple layer approach, since one zone would include the information of several RATs and cells, it is expected that $M$ will be larger than with the single layer approach, thus increasing even more the demanded CPC capacity.

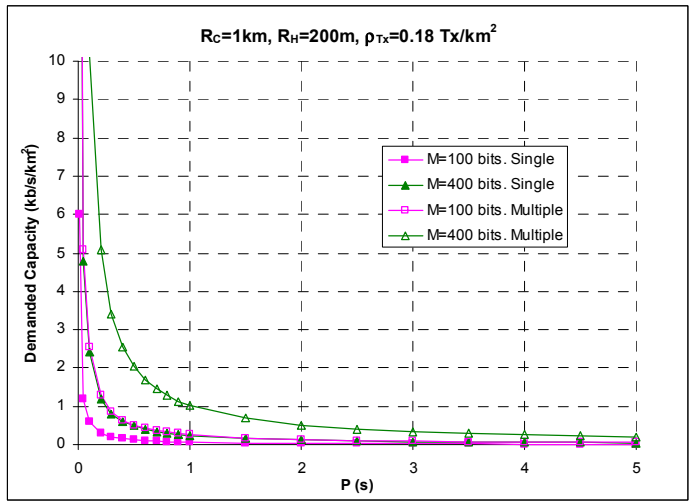

Figure 10. Comparison between the single and the multiple layer case for the broadcast CPC in scenario 1.

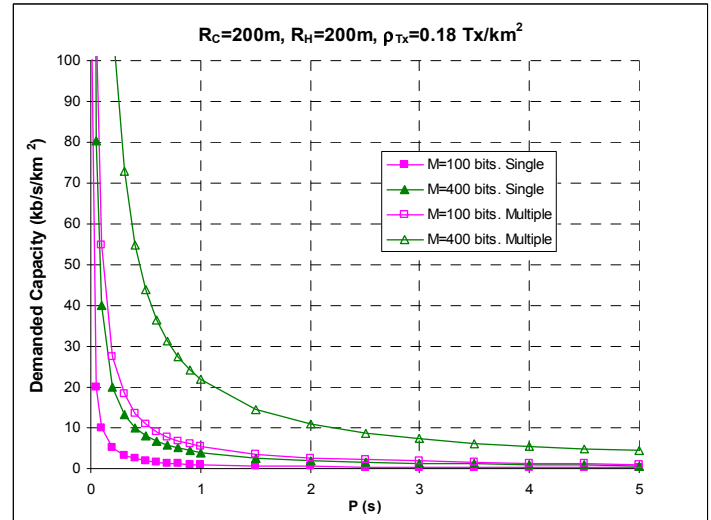

Figure 11. Comparison between the single and the multiple layer case for the broadcast CPC in scenario 3.

\section{CPC dimensioning}

Once the demanded capacity has been computed the adequate values of the density of CPC transmitters $T$ $\left(\mathrm{TRx} / \mathrm{km}^{2}\right)$ and the bit rate per transmitter $R(\mathrm{~b} / \mathrm{s} / \mathrm{TRx})$ should be dimensioned, so that the product $R \times T$ equals the demanded capacity. In this respect, Figure 12 illustrates for different required capacities the existing degree of freedom between the number of CPC transmitters and the bit rate available at the radio interface. If $R$ is lower, then higher $T$ is needed, and vice versa.

As an example, let assume scenario 1 with the broadcast approach, $P=1 \mathrm{~s}$ and $M=400$ bits. From Figure 6, the requested capacity with the multiple layer case is around $1 \mathrm{~kb} / \mathrm{s} / \mathrm{km}^{2}$. In this case, one possible deployment of the CPC would be using the cellular infrastructure existing in the scenario, corresponding to a CPC transmitter density of $T=0.42$ $\mathrm{TRx} / \mathrm{km}^{2}$ (this is computed as the number of cells in the scenario divided by the total surface of $100 \mathrm{~km}^{2}$ ) Under these conditions, from Figure 12 the corresponding bit rate requirement would be around $2.4 \mathrm{~kb} / \mathrm{s} / \mathrm{TRx}$. In a short term CPC solution, this capacity could most probably be obtained 
using e.g. sparing bits from BCCH in GSM and, therefore, CPC could be implemented as an in-band solution by reusing existing legacy GSM infrastructure and being on an already allocated GSM frequency.

As a further illustration, let assume that, for the same scenario, the requirement is set to $P=0.2 \mathrm{~s}$ instead of $P=1 \mathrm{~s}$ considered before. In this case, according to Figure 6 the requested capacity would rise up to around $5 \mathrm{~kb} / \mathrm{s} / \mathrm{km}^{2}$. Then, if it is intended to reuse the cellular infrastructure to deploy CPC transceivers, i.e. $T=0.42 \mathrm{TRx} / \mathrm{km}^{2}$, the required capacity per transmitter $R$, obtained from Figure 12, would rise up to around $12 \mathrm{~kb} / \mathrm{s}$. This capacity could be hardly obtained from sparing bits of a GSM BCCH channel. In this case, an ondemand $\mathrm{CPC}$ solution could be favored since it would require $1 \mathrm{~kb} / \mathrm{s} / \mathrm{km}^{2}$ if the rate of requests $L$ is below $2.5 \mathrm{reqs} / \mathrm{s} / \mathrm{km}^{2}$ (see Figure 9). In this way, the required CPC data rate in downlink per transmitter would be lowered to $R=2.4 \mathrm{~kb} / \mathrm{s}$.

Finally, let consider scenario 2, with $P=0.1 \mathrm{~s}$, the multiple layer case and $M=400$ bits. The requested CPC capacity would be, according to Figure 7 , close to $15 \mathrm{~kb} / \mathrm{s} / \mathrm{km}^{2}$. In this case, reusing the cellular infrastucture with $0.42 \mathrm{TRx} / / \mathrm{km}^{2}$ would need a total of around $35 \mathrm{~kb} / \mathrm{s}$. On a long term perspective, this capacity could be provided e.g. by an out-band solution, through a newly identified access technology potentially transmitted on a harmonized frequency band.

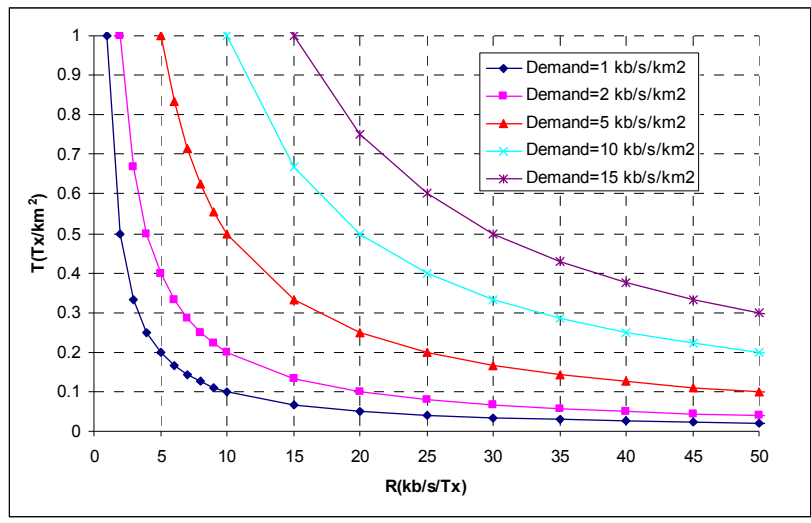

Figure 12. Trade-off between the CPC transmitter density and the CPC transmitter bit rate for different demands.

\section{CONCLUSIONS}

This paper has considered a CPC solution as a mechanism allowing the terminal to be aware of the communication means available at a given time and place in a flexible spectrum management framework, where a number of transceivers with flexible time-varying assignment of operating frequency and/or RAT are deployed. The motivations behind considering a CPC solution instead of a sensing-based approach is sustained on the presumption that spectrum sensing may result in a very time- and powerconsuming operation if the spectrum bands to be sensed are too large.

The paper has highlighted different aspects to be considered when moving from CPC concept to implementation and deployment, identifying different options related to the RAT used for CPC (In-band, Out-band), information delivery mode (Broadcast, On-demand), information structuring scheme (Single layer, Multiple layer), etc. As a result, a CPC dimensioning methodology has been formulated, allowing a clear identification of the main parameters involved in the process and, therefore, facilitating the identification of design trade-offs. Therefore, this paper has provided a rather generic framework for benchmarking the most suitable CPC implementation solution for a given scenario.

In order to illustrate that no single CPC implementation solution can be anticipated as the most suitable solution in all possible scenarios, three illustrative scenarios have been presented and analyzed. Thus, while an "in-band" "broadcast" "single-layer" CPC reusing existing GSM infrastructure can be a valid, convenient and simple solution in several practical scenarios, this could not be so feasible if strong requirements on the maximum allowed delay in retrieving CPC information are set. In such case, for example, an "on-demand" instead of "broadcast" approach could extend its feasibility.

\section{ACKNOWLEDGEMENTS}

This work was performed in project $\mathrm{E}^{3}$ which has received research funding from the Community's Seventh Framework program. This paper reflects only the authors' views and the Community is not liable for any use that may be made of the information contained therein. The contributions of colleagues from $\mathrm{E}^{3}$ consortium are hereby acknowledged. This work has also been supported by the Spanish Research Council and FEDER funds under COGNOS grant (ref. TEC2007-60985).

\section{REFERENCES}

[1] D. Bourse et al., "The $E^{2} R$ II Flexible Spectrum Management (FSM) Framework and Cognitive Pilot Channel (CPC) Concept - Technical and Business Analysis and Recommendations", E ${ }^{2} \mathrm{R}$ II White Paper, November 2007.

[2] Federal Communications Commission (FCC), "Notice of Proposed Rule Making," ET Docket no. 04-113, May 25, 2004.

[3] M. Buddhikot, P. Kolodzy, S. Miller, K. Ryan, J. Evans “ DIMSUMNet: New Directions in Wireless Networking Using Coordinated Dynamic Spectrum Access", IEEE International Symposium on a World of Wireless, Mobile and Multimedia Networks (IEEE WoWMoM), Taormina/Giardini Naxos, Italy, June, 2005.

[4] D. Raychaudhuri, X. Jing "A Spectrum Etiquette Protocol for Efficient Coordination of Radio Devices in Unlicensed Bands", 14th IEEE 2003 International Symposium on Persona1, Indoor and Mobile Radio Communication Proceedings (PIMRC), Beijing, September, 2003.

[5] P. Houzé, S. Ben Jemaa, P. Cordier "Common Pilot Channel for Network Selection", IEEE VTC in Spring Conference, Melbourne, May, 2006.

[6] P. Cordier et al., "E2R Cognitive Pilot Channel concept", IST Summit, Mykonos, June, 2006.

[7] J. Pérez-Romero, O. Sallent, R. Agustí, L. Giupponi, "A Novel OnDemand Cognitive Pilot Channel enabling Dynamic Spectrum Allocation”, DySPAN ‘07, Dublin, April, 2007.

[8] IEEE P1900.4, Draft Standard for Architectural building blocks enabling network-device distributed decision making for optimized radio resource usage in heterogeneous wireless access networks.

[9] J. Pérez-Romero, O. Sallent, R. Agustí, "On the Applicability of Image Processing Techniques in the Radio Environment Characterisation", IEEE Vehicular Technology Conference in Spring (VTC-2009), Barcelona, April 2009. 